\title{
Calidad de vida, hábitos alimenticios y adherencia al tratamiento de los pacientes con VIH/SIDA
}

\section{Quality of life, eating habits and adherence to the treatment of HIV-AIDS patients}

\author{
Lilia Azucena Romero Sacoto ${ }^{1 *}$ y Andrés Alexis Ramírez Coronel ${ }^{1,2}$ \\ ${ }^{1}$ Universidad Católica de Cuenca \\ 2 Laboratorio de Psicometría y Neurociencias Cognitivas \\ *lromeros@ucacue.edu.ec
}

DOI: https://doi.org/10.26871/killkana_salud.v3i3.528

\begin{abstract}
Resumen
Contexto: La calidad de vida es la percepción que un individuo tiene de su lugar en la existencia, en el contexto de la cultura y del sistema de valores en los que vive y en relación con sus objetivos, sus expectativas, sus normas, sus inquietudes. Objetivo: En objetivo principal es determinar la calidad de vida, hábitos alimenticios y adherencia al tratamiento de los pacientes con VIH/SIDA del Hospital Homero Castanier Crespo-Azogues. Procedimiento: Se realizó un estudio cuantitativo, descriptivo de corte transversal de una muestra de 32 paciente diagnosticados VIH/SIDA de la Provincia del Cañar. Para la selección de los pacientes se realizó un muestreo no probabilístico-aleatorio. Se utilizaron el cuestionario para medir una encuesta sociodemográfica, cuestionario SMAQ para medir adherencia, escala de calidad de vida y cuestionario sobre el estado nutricional. Se efectuó un análisis descriptivo las variables de los factores sociodemográficos y las variables de estudio. Posteriormente se verificó asociaciones mediante la prueba de Chi-cuadrado entre las variables sociodemográficas con la calidad de vida, adherencia al tratamiento y el estado nutricional, para los análisis se usó el estadístico SPSS 26. Resultados: Se presentó adherencia al trabamiento $(56 \%)$, mala calidad de vida $(53,1 \%)$ y nivel nutricional bajo $(53,1 \%)$. En las correlaciones entre calidad de vida y nivel nutricional $\mathrm{p}=0,047$, estado de salud general y calidad de vida percibida $\mathrm{p}=0,002$ y adherencia al tratamiento y salud mental del paciente $\mathrm{p}=0,033$. Conclusiones: La mitad de la población estudiada presenta mala calidad de vida, adherentes al tratamiento y nivel nutricional bajo. La calidad de vida está asociada positivamente con el estado nutricional y estado de salud general, y se presentó una relación entre adherencia y salud mental del paciente.
\end{abstract}

Palabras clave: Calidad de vida, nivel nutricional, adherencia VIH/SIDA.

\begin{abstract}
Context: The quality of life is the perception that an individual has of his place in existence, in the context of the culture and value system in which he lives and in relation to his objectives, his expectations, his rules, your concerns. Objective: The main objective is to determine the quality of life, eating habits and adherence to treatment of patients with HIV-AIDS at the Homero Castanier Crespo-Azogues Hospital. Procedure: A quantitative, descriptive cross-sectional study of a sample of 32 patients diagnosed with HIV-AIDS in the Province of Cañar was carried out. For the selection of patients, a non-probabilistic-random sampling was performed. The questionnaire was used to measure a sociodemographic survey, $S M A Q$ questionnaire to measure adherence, quality of life scale and questionnaire on nutritional status. A descriptive analysis was made of the variables of the sociodemographic factors and the study variables. Subsequently, associations were verified using the Chi-square test between sociodemographic variables with quality of life, adherence to treatment and nutritional status, for the analysis the SPSS 26 statistic was used. Results: Adherence to work was presented (56 \%), poor quality of life (53.1 \%) and low nutritional level (53.1\%). In the correlations between quality of life and nutritional level $p=0.047$, general health status and perceived quality of life $p=0.002$ and adherence to treatment and mental health of the patient $p=0.033$. Conclusions: Half of the population studied has poor quality of life, adherents to treatment and low nutritional level. Quality of life is positively associated with nutritional status and general health status, and a relationship between adherence and mental health of the patient was presented.
\end{abstract}

Keywords: Quality of life, nutritional level, HIV-AIDS adherence. 


\section{Introducción}

El Virus de Inmunodeficiencia Humana (VIH), es el responsable de la destrucción de los linfocitos CD4. Estas células forman parte del sistema inmunitario y se encargan de la organización y coordinación de la respuesta inmune de organismo contra las infecciones. EL ARN del virus se copia al ADN de las células, las infecta y se integra a su genoma, parasita las células y destruye el sistema inmunológico, y deja al individuo propenso a sufrir todo tipo de infecciones.

El VIH/SIDA, es uno de los más graves problemas de salud pública en el mundo, especialmente en los países en desarrollo. Según la Organización Mundial de la Salud (OMS) en el año 2017, hubo un total de 36,7 millones de personas infectadas por el VIH, personas vulnerables que fueron afectadas por la epidemia. La OMS trabaja para mejorar el acceso universal a servicios de atención sanitaria, medicamentos, medios diagnósticos y productos básicos para la salud; con el acceso al tratamiento antirretroviral, las personas seropositivas pueden mejorar su estado de salud y prolongar su vida. En el año 2017 se determinó que alrededor de 20,9 millones de personas recibieron la terapia antirretroviral en todo el mundo. ${ }^{1}$

En Ecuador, el Ministerio de Salud Pública (MSP) reporta 39,224 personas que viven con VIH, de ellas menos de la mitad, el $43 \%$, (16.997) recibieron el tratamiento. Ecuador se encuentra entre los cinco países de América Latina con más contagios de VIH desde los años 2005 hasta el $2015 .^{2}$

La epidemia de VIH/SIDA en Ecuador se ha concentrado en personas transfemeninas (MTF) $(34,8 \%$ en Quito y $20,7 \%$ en Guayaquil) y de hombres que tienen sexo con hombres (HSH) (16,5\% en Quito y $11,2 \%$ en Guayaquil). Es importante mencionar que en la población general la prevalencia nacional es de 0,3 el grupo de edad más afectado esta entre 15 y 49 años. La prevalencia para el año 2017 está en la población sexualmente activa con edad entre 1549 años. En el año 2017 se pronosticó en Ecuador una tasa de personas viviendo con VIH/SIDA de 0,22 por cada 1.000 habitantes.

La trasmisión materno infantil del VIH puede darse durante el embarazo, en el momento del parto o a través de la lactancia materna. En el año 2017, se continúa con la estrategia para la eliminación de la trasmisión materno infantil. Los niños o niñas cuyas madres son VIH positivo, no necesariamente se infectarán durante el nacimiento, se evitará la infección si se consideran las directrices señaladas por la estrategia y se reduce el riesgo de infección del $40 \%$ al $2 \%$. La carga viral de la madre es el principal factor de riesgo en la trasmisión materno infantil, a mayor carga viral de la madre el riesgo de trasmisión al niño es mayor. La estrategia para disminuir el riesgo es la administración de antirretrovirales a la madre lo antes posible.

Ecuador presenta una prevalencia de VIH en mujeres embarazadas de 0,16. La provincia del Guayas se presenta la mayor concentración de notificación de casos nuevos. En 2017 la provincia con mayor número de casos reportados es el Guayas, con el $31 \%$, seguido por Pichincha con $23 \%$, Esmeraldas con $7 \%$, El Oro con 5\%, Los Ríos y Manabí con 4,9\%. ${ }^{3}$ En Ecuador se registraron un total de 17.574 defunciones por SIDA. En la provincia del Cañar en el año 2016 un total de 39 casos y desde el año de 1984 hasta la fecha, se reportan un total de $263(0,4 \%)$ casos. La incidencia es de 1,48 por cada 10.000 habitantes. $^{4}$

Se considera como calidad de vida el grado en el que un individuo conserva su salud, participa e interactúa con el medio donde este se desarrolla. El término calidad de vida es intrínsecamente ambiguo, porque puede ser parte de la experiencia de la vida de los individuos y las condiciones de vida en que se encuentran las personas. En consecuencia, la calidad de vida es muy subjetiva. La calidad de vida se mide por la satisfacción con la vida, y la adquisición de capacidades (por ejemplo, tener la capacidad de vivir una buena vida en términos de bienestar emocional y físico). Las personas con discapacidad pueden reportar una alta calidad de vida, personas sanas que presentan problemas económicos pueden reportar una baja calidad de vida. Desde esta perspectiva el cuidado de la salud, la calidad de vida se considera multidimensional, y abarca el bienestar emocional, físico, material y social. ${ }^{5}$

El objetivo de la investigación fue evaluar la calidad de vida, el estado nutricional y la adherencia al tratamiento de las pacientes con VIH/SIDA atendidos en el hospital Homero Castanier Crespo de la ciudad de Azogues.

\section{Antecedentes}

El Art. 32 de la Constitución de la República del Ecuador establece que: "la salud es un derecho que garantiza el estado, cuya realización se vincula al ejercicio de otros derechos, entre ellos el agua, la alimentación, la educación, la cultura física, el trabajo, la seguridad social, los ambientes sanos y otros que sustentan el buen vivir". El cumplimiento de este derecho es fundamental en todos los ecuatorianos, está inmerso el derecho a una calidad de vida digna, sin ningún tipo de estigmas.

El Sistema Nacional de Salud, brinda servicios que se orientan a mejorar la calidad de vida de la población en general. Estos servicios están amparados en los principios de: universalidad, equidad, solidaridad, calidad, eficiencia, eficacia, con enfoque de género y generacional, respetando la interculturalidad. En este sentido se han creado políticas, programas y proyectos orientados a los servicios de promoción, prevención y atención integral de salud sexual y reproductiva.

Bajo estas premisas y el amparo legal con el que cuenta el Sistema Nacional de Salud se garantiza una atención eficiente, eficaz, con calidad y calidez a todos los grupos vulnerables de la población, siendo uno de ellos los pacientes con VIH/SIDA que se atienden en el hospital Homero Castanier Crespo.

Mejorar la calidad de atención de la población vulnerable es el objetivo principal del Sistema Nacional de Salud, sin duda que dentro de este grupo están los pacientes que 
viven con VIH/SIDA. Por esta razón en el año 2002 se crea el Reglamento de Atención a Personas con VIH/SIDA, según acuerdo ministerial 732, Reg. Oficial 739. Los objetivos están en relación a brindar una atención especializada, estandarizar los protocolos de atención, disminuir la contaminación vertical y vigilar el cumplimiento de los derechos de las personas con VIH/SIDA, lo que permite el acceso permanente y oportuno a los servicios de salud.

El Ministerio de Salud Pública del Ecuador, mediante el Sistema Nacional de Salud garantiza la atención gratuita, realización de exámenes de laboratorio y el tratamiento específico y complementario de los diversos grupos de población que padecen VIH/SIDA.

El Reglamento de atención a personas con VIH/SIDA menciona que se les pueden suspender los beneficios del programa cuando se incumpla el Reglamento. En consecuencia, es muy importante la promoción de los servicios que brinda el Sistema Nacional de Salud del Ecuador, sobre todo la atención dirigida a los grupos de población vulnerable como las personas viviendo con VIH/SIDA. Todo ello ligado a lo establecido en el Plan Nacional de Desarrollo "TODA UNA VIDA". Eje 1. "Derechos para todos durante toda la vida". Objetivo 1. "Garantizar una vida digna con iguales oportunidades para todas las personas"

El concepto de calidad de vida (CDV) en los últimos años ha adquirido relevancia sobre todo cuando se trata de evaluar la calidad de vida de grupos vulnerables, como es el caso de las personas que viven con VIH/SIDA. Las investigaciones realizadas demuestran que la calidad de vida relacionada con salud de los pacientes vulnerables puede verse afectada por múltiples factores, ello ha originado el interés de los investigadores de conocer la calidad de vida de los pacientes que viven con VIH/SIDA.

Los factores asociados a la calidad de vida relacionada con salud de las personas que viven con VIH/SIDA fue estudiado en Uruguay por Cabrera y col. en el año 2018. Trabajaron con una muestra de 101 pacientes a los cuales se les aplicó el cuestionario MOS-HIV SF 30 y el cuestionario SMAQ para evaluar adherencia al tratamiento. Los resultados fueron: la encuesta MOS-HIV mostró fiabilidad y consistencia interna, el Score global de CVRS fue de $71.71 \pm 17,76$, en las personas con carga viral indetectable el score fue malo, funcionamiento físico: $\mathrm{p}=0.016$, energía y fatiga $\mathrm{P}=0,008$, calidad de vida percibida $\mathrm{p}=0,025$.

En mujeres el score más bajo fue en dolor $\mathrm{p}=0.034$, funcionamiento físico $\mathrm{p}=0.006$, funcionamiento social $\mathrm{p}=$ 0.031 , salud mental $\mathrm{p}=0.040$, el score fue mejor en los pacientes adherentes, salud general $\mathrm{p}=0.027$. Se concluye que a pesar de que se trata de una población muy vulnerables el score ha sido bueno. La adherencia al tratamiento se relacionó con alguna dimensión de la encuesta, pero no tuvo impacto sobre el score global. ${ }^{8}$

Frutos C y col. en el año 2015 en Paraguay estudiaron la calidad de vida de los pacientes con VIH, se trabajó con una muestra de 329 pacientes. Fue un estudio observacional, descriptivo de corte transversal, retrospectivo.
Como instrumento de recolección de datos se utilizó la encuesta WHOQOL-HIV-BREF. Los resultados fueron los siguientes: en relación a las características sociodemográficas $53 \%$ fueron del sexo masculino, $52 \%$ solteros, $59 \%$ tenían instrucción secundaria, $47 \%$ han sido discriminados, en cuanto a la calidad de vida existía un rango entre 66 y 79 de calificación intermedia de buena. ${ }^{9}$

La calidad de vida y la variable psicológica que afectan la adherencia al tratamiento antirretroviral en pacientes mexicanos con infección por VIH/SIDA, fue estudiado por Itzel $\mathrm{G}$ y col. en un estudio comparativo, analítico, transversal que se realizó en el año 2017 y con una muestra de 160 pacientes. Su usaron como instrumentos las encuestas MOS SF 16 y VPAD 24. Los resultados fueron: en relación a las características demográficas: $86.5 \%$ sexo masculino, edad promedio 38.8 años, el $53.3 \%$ con orientación sexual HSH, con más o menos 7 años de diagnóstico de VIH/SIDA, el $65 \%$ de los pacientes se adhirió al tratamiento, $61 \%$ de pacientes tuvo conocimiento de la enfermedad, $63 \%$ de los pacientes presentó comportamiento depresivo, 30,6\% presenta adicción al alcohol o alguna droga, durante el análisis de las dimensiones de la calidad de vida se observó que un $97.2 \%$ de los pacientes se ubicó en el puntaje de 51 a 100 (mejor calidad de vida). La adherencia al tratamiento estuvo asociada con el comportamiento depresivo, el cual estuvo asociado con las adicciones. Se concluye que la buena adherencia al TAR estuvo asociada a evitar el comportamiento depresivo, y a la ausencia de adicciones y no se asoció a la calidad de vida. ${ }^{10}$

El objetivo de la enfermería es el cuidado al ser humano en toda su integridad, ello incluye su entorno. La calidad de vida de los pacientes con VIH/SIDA y la atención integral de enfermería en Paraguay en el año 2010 realizaron una investigación cuantitativa, descriptiva, observacional transversal, y se estudió una muestra de 50 pacientes. Se encontró lo siguiente: predominó el sexo masculino, la edad promedio fue de 20-30 años, y el nivel socioeconómico era bajo. Los signos y síntomas que presentaron los pacientes fueron: náuseas, vómitos, diarrea, disfagia, odinofagia, convulsiones, agotamiento físico, lesiones cutáneas. Las infecciones oportunistas presentes fueron: Síndrome de Wasting, anemia, meningitis por Cryptococcus, diarrea y deshidratación. La calidad de vida dimensión energía, salud mental, dimensión emocional, función cognitiva, y calidad de interacción social alcanzaron una puntuación muy baja. Los pacientes calificaron como muy buena y excelente la atención brindada en el centro. ${ }^{11}$

En Brasil se realiza un estudio para conocer la calidad de vida de personas conviviendo con HIV/SIDA: relación temporal, sociodemográfica y perceptiva de la salud, fue un estudio cuantitativo transversal. Se estudiaron a 100 personas seropositivas, se recolectaron datos sociodemográficos y para medir la calidad de vida se usó la encuesta WHOQOL-HIV-BREF, los datos se analizaron en estadísticos descriptivos e inferencial. Los resultados fueron: la percepción de la calidad de vida fue intermedia en todos los 
dominios de la calidad de vida. Se identificó relación entre mayor satisfacción con salud y mejor calidad de vida, así como diferencias estadísticamente significativas entre dimensiones de la calidad de vida según género, situación de empleo, renta familiar, renta personal, orientación religiosa y tiempo de diagnóstico. ${ }^{12}$

\section{Metodología}

Estudio cuantitativo, descriptivo de corte transversal. Se trabajó con una muestra censal de 32 de 39 pacientes con diagnóstico de VIH/SIDA en la provincia del Cañar. Por tratarse de población vulnerable se tomaron las siguientes consideraciones éticas:

Criterios de inclusión: Se incluyeron todos los pacientes con diagnóstico de VIH/SIDA que acuden al servicio de consulta externa del Hospital Homero Castanier Crespo, mayores de 18 años que firmen el consentimiento informado.

Criterios de Exclusión: Pacientes con diagnóstico de VIH/SIDA menores de 18 años, pacientes que no acepten participar en la investigación o que luego de haber firmado el consentimiento informado no quieran llenar los cuestionarios.

La investigación se realizó cumpliendo las normas de Helsinki para la investigación en seres humanos.

\subsection{Métodos, técnicas e instrumentos de recolección de datos}

Método: Durante la aplicación del instrumento el investigador permaneció en el consultorio, al momento del ingreso del paciente y en colaboración con el médico tratante se le invitó a participar en la investigación. Una vez que el paciente aceptó participar fue llevado a un consultorio que aseguró la privacidad y discreción, se le brindó información acerca de las encuestas y luego de la obtención del consentimiento informado se aplicaron las encuestas.

Técnica: Encuesta.

Instrumento: cuestionario. Se utilizaron 4 cuestionarios:

Los factores sociodemográficos: Fueron valorados y con el uso de un cuestionario que midió: edad, sexo, estado civil, nivel de instrucción, residencia, situación laboral, consumo de sustancias y enfermedades adicionales. La aplicación de la encuesta tuvo una duración de 2 minutos.

\subsection{Cuestionario MOS-HIV SF 30}

La calidad de vida en pacientes con VIH/SIDA se midió con la encuesta MOS-HIV SF 30 que deriva de la encuesta MOS-HIV consta de 35 ítems. Esta encuesta es el instrumento específico que se utiliza para evaluar la calidad de vida en pacientes con VIH/SIDA. La encuesta MOS HIV SF 30 es una versión corta que se encuentra validada en español por Remor. ${ }^{12} \mathrm{El}$ instrumento tiene 11 dimensiones, con 30 ítems que indagan sobre: Estado de salud general, dolor, actividad diaria, funcionamiento físico, funcionamiento social, salud mental, energía/fatiga, malestar respecto a la salud, funcionamiento cognitivo, calidad de vida percibida, y transición de salud. El instrumento fue aplicado en promedio 10 minutos por cada encuesta. A pesar de un sin número de instrumentos genéricos como específicos, los únicos cuestionarios que se encuentran validados y traducidos al español para evaluar a pacientes con VIH son el MOS HIV y el MQOL- HIV. El MOSHIV SF son instrumentos que han demostrado fiabilidad y validez en estudios anteriores, por ello se decidió utilizar el cuestionario.

La encuesta MOS HIV contiene de 11 dimensiones; y son valoradas en base a rangos, que van de 0-4. Estas son: salud general percibida, dolor, actividad diaria, funcionamiento social, calidad de vida percibida y transición de salud. La dimensión funcionamiento físico, su rango está entre 0-12; y de 0-16 están las dimensiones energía-fatiga, malestar respecto a la salud y funcionamiento cognitivo, y de 0-20 está la salud mental. En consecuencia, una vez realizada la sumatoria global, se considera como buena calidad de vida aquellos pacientes que se ubican dentro del rango de 71-100, mientras que rangos inferiores a 71 se consideran como mala calidad de vida.

\subsection{Cuestionario de adherencia al tratamiento}

En los pacientes que viven con VIH/SIDA la adherencia al tratamiento es fundamental. Según la literatura revisada, es un indicador de calidad de vida, en el grupo de pacientes estudiados, y se utilizó el cuestionario de adherencia SMAQ ( Simplified Medication Adherence Questionnaire). Este cuestionario es un instrumento que fue traducido al español quese emplea para medir la adherencia a un tratamiento. Tiene seis preguntas las cuales cuatro son cualitativas y dos son cuantitativas. La interpretación se realiza de la siguiente manera: el paciente es no adherente cuando las respuestas a las preguntas 1 y 3 son afirmativas, cuando la pregunta 2 es negativa, la pregunta 4 cualquier respuesta diferente a ninguna se considera no adherente.

La pregunta 6 se refiere a cuántos días no ha tomado su medicación correctamente, si la respuesta es más de dos veces se considera al paciente como no adherente. La pregunta 5 del cuestionario hace referencia a si el paciente se ha olvidado de tomar su medicación, si la respuesta es afirmativa se considera como no adherente. La aplicación de la encuesta tuvo una duración de 5 minutos.

\subsection{Test KRECE PLUS}

El test fue validado por (Serra \& Aranceta, Desayuno y equilibrio alimentario estudio en kids, 2010) consta de 16 ítems, cada uno tiene una valoración de +1 o -1 . La máxima puntuación es +11 y -5 la mínima puntuación. De la puntuación total obtenida se encasillan en tres fases: nivel nutricional alto, nivel nutricional medio, nivel nutricional bajo. Cuando se obtiene una puntuación mayor o igual a 9 se obtiene un nivel nutricional alto, de 6 a 8 nivel nutricional medio, y una puntuación igual o menor a 5 nivel nutricional bajo. El tiempo de aplicación del test es de 5 minutos. 
Los datos recopilados se clasificaron y agruparon de acuerdo a las variables de estudio. Para la presentación se realizó en el siguiente orden: Validación y Edición, Codificación, introducción de datos, tabulación y análisis estadísticos. Se utilizó como medida el porcentaje en el programa IBM Statistics SPSS versión 23. Los datos luego de su análisis se organizaron en tablas de frecuencias agrupando en clases o categorías de respuestas de donde se obtuvieron los datos cuantitativos. La relación entre variables se realizó mediante las pruebas del Chi cuadrado, por otra parte, para valorar la significancia estadística se consideró un nivel de significación de 0,5 .

\section{Resultados}

Tabla 1. Variables sociodemográficas de los pacientes con VIH/SIDA

\begin{tabular}{lcc}
\hline Variable & Frecuencia & Porcentaje \\
\hline Edad & & \\
$18-29$ & 9 & 29 \\
$30-39$ & 16 & 50.6 \\
$40-49$ & 3 & 9.7 \\
$50-60$ & 3 & 9.7 \\
\hline Residencia & & \\
Urbano & 19 & 59 \\
Rural & 13 & 40.6 \\
\hline Estado Civil & & \\
Soltero & 18 & 56.3 \\
Casado & 7 & 21.9 \\
Divorciado & 2 & 6.3 \\
Unión Libre & 4 & 12.5 \\
Viudo & 1 & 9.1 \\
\hline Género & & \\
Masculino & 21 & 65.6 \\
Femenino & 11 & 34.4 \\
\hline Instrucción & & \\
Primaria & 15 & 46.9 \\
Secundaria & 9 & 28.1 \\
Superior & 8 & 25 \\
\hline
\end{tabular}

Fuente: Los autores

Las características sociodemográficas de los pacientes estudiados demuestran que el $50.6 \%$ de los pacientes está en un grupo de edad de entre 30 y 39 años. En su mayoría (59\%) proceden del área urbana, de estado civil solteros, el $56.3 \%$; en cuanto al género el $65.6 \%$ son hombres y de acuerdo al nivel de instrucción el $46.9 \%$ apenas tiene la instrucción primaria.

Tabla 2. Orientación sexual de los pacientes con VIH/SIDA

\begin{tabular}{lcc}
\hline Variable & Frecuencia & Porcentaje \\
\hline Homosexual & 2 & 6.3 \\
Heterosexual & 27 & 84.4 \\
Bisexual & 2 & 6.3 \\
Transexual & 1 & 3.1 \\
Total & 32 & 100 \\
\hline
\end{tabular}

Fuente: Los autores

Es importante conocer la orientación sexual del grupo estudiado. La encuesta dividió la orientación sexual en 4 tipos: homosexual, heterosexual, bisexual y transexual. El estudio revela que de los 32 pacientes estudiados el $84.4 \%$ son heterosexuales, y apenas el $3.1 \%$ son transexuales.

Tabla 3. Consumo de sustancias estupefacientes

\begin{tabular}{lcc}
\hline Variable & Frecuencia & Porcentaje \\
\hline Alcohol & 10 & 31.3 \\
Tabaco & 6 & 18.8 \\
Ninguna & 16 & 50 \\
TOTAL & 32 & 100 \\
\hline
\end{tabular}

Fuente: Los autores

El consumo de otras drogas y alcohol en los pacientes con VIH/SIDA son comportamientos de riesgo que multiplican la probabilidad de infectarse, coinfecciones o trasmisión de las mismas, consumir drogas ajenas al tratamiento antirretroviral puede interrumpir el cumplimiento del régimen terapéutico diario y no permitir la adherencia al tratamiento. Los resultados del estudio revelan que el $50 \%$ de los pacientes encuestados no consumen ningún tipo de drogas, sin embargo, un porcentaje importante, el $31.3 \%$ dice consumir alcohol, y el $18.8 \%$ fuma cigarrillo.

Tabla 4. Adherencia al tratamiento

\begin{tabular}{lcc}
\hline Variable & Frecuencia & Porcentaje \\
\hline Adherente & 18 & 56.3 \\
No adherente & 14 & 43.8 \\
TOTAL & 32 & 100 \\
\hline
\end{tabular}

Fuente: Los autores

La esperanza de vida en los pacientes con VIH en la actualidad está en crecimiento, puesto que se ha catalogado como una enfermedad crónica. En los pacientes mayores de 40 años seropositivos se presentan las mismas enfermedades crónicas semejantes en la de las personas seronegativas. Sin embargo, la adherencia al tratamiento resulta fundamental para mantener en crecimiento la esperanza de vida. En la tabla se observa que el $56,3 \%$ de los pacientes son adherentes al tratamiento, y el $43.8 \%$ de los pacientes no son adherentes.

Tabla 5. Calidad de vida

\begin{tabular}{lcc}
\hline Variable & Frecuencia & Porcentaje \\
\hline Adherente & 18 & 56.3 \\
No adherente & 14 & 43.8 \\
TOTAL & 32 & 100 \\
\hline
\end{tabular}

Fuente: Los autores

Se valoró la calidad de vida según la encuesta MOS HIV SF 30. La puntuación obtenida mediante la suma del score de cada dimensión indican que más de la mitad, el $53,1 \%$ de los encuestados tienen una mala calidad de vida mientras que el $46,9 \%$ de los encuestados calificó como buena calidad de vida. 
Tabla 6. Nivel Nutricional

\begin{tabular}{lcc}
\hline Variable & Frecuencia & Porcentaje \\
\hline Nivel nutricional alto & 3 & 9.4 \\
Nivel nutricional medio & 12 & 37.5 \\
Nivel nutricional bajo & 17 & 53.1 \\
\hline
\end{tabular}

Fuente: Los autores

El instrumento utilizado Krece Plus, valora hábitos alimenticios y clasifica el nivel nutricional del paciente a través del análisis de la principal comida del día, el desayuno y equilibrio alimentario. De los 32 pacientes que reciben atención en el hospital Homero Castanier Crespo, más de la mitad $(53.1 \%)$ tienen un nivel nutricional bajo a sabiendas de que su condición clínica obliga a mantener un nivel nutricional y estado nutricional adecuado.

Tabla 7. Calidad de vida y el nivel nutricional

\begin{tabular}{lcccc}
\hline $\begin{array}{l}\text { Calidad } \\
\text { de vida }\end{array}$ & $\begin{array}{c}\text { Nivel } \\
\text { nutricional } \\
\text { alto }\end{array}$ & $\begin{array}{c}\text { Nivel } \\
\text { nutricional } \\
\text { medio }\end{array}$ & $\begin{array}{c}\text { Nivel } \\
\text { nutricional } \\
\text { bajo }\end{array}$ & TOTAL \\
\hline Buena & 3 & 7 & 5 & 15 \\
Mala & 0 & 5 & 12 & 17 \\
Total & 3 & 12 & 17 & 32 \\
\hline
\end{tabular}

Fuente: Los autores

El nivel nutricional de los pacientes estudiados se midió utilizando el test rápido de Krece plus que consta de 16 ítems, cada uno con una valoración de más 1 o menos 1 . En dependencia de la puntuación total, el nivel nutricional se clasifica como: nivel nutricional alto, medio o bajo. En la tabla de relación entre estas dos variables se observa que 12 de los pacientes tienen una mala calidad de vida y un nivel nutricional bajo. Se aplica la prueba de Chi cuadrado y se obtiene significancia estadística $\mathrm{p}=0.047$.

Tabla 8. Calidad de vida y salud general percibida

\begin{tabular}{lcccc}
\hline $\begin{array}{l}\text { Calidad } \\
\text { de vida }\end{array}$ & Muy buena & Buena & Regular & Mala \\
\hline Buena & 5 & 10 & 0 & 0 \\
Mala & 2 & 4 & 9 & 2 \\
TOTAL & 7 & 14 & 9 & 2 \\
\hline
\end{tabular}

Fuente: Los autores

Según el instrumento aplicado a los pacientes se determinó que el $53.1 \%$ de los encuestados tiene una mala calidad de vida, el sumatorio total de los rangos dio valores inferiores a 71 en más de la mitad de los pacientes. Se buscó la relación entre la salud general percibida por el paciente y su calidad de vida mediante la prueba de Chi cuadrado y se encontró que existe significancia estadística, el valor de $\mathrm{p}=0.002$.
Tabla 9. Adherencia al tratamiento y salud mental del paciente

\begin{tabular}{lccc}
\hline Salud Mental & Adherente & $\begin{array}{c}\text { No } \\
\text { adherente }\end{array}$ & TOTAL \\
\hline Excelente & 7 & 1 & 8 \\
Muy buena & 8 & 4 & 12 \\
Buena & 3 & 8 & 11 \\
Regular & 0 & 1 & 1 \\
TOTAL & 18 & 14 & 32 \\
\hline
\end{tabular}

Fuente: Los autores

La salud mental y la adherencia al tratamiento son dimensiones que mide el instrumento utilizado. La salud mental del paciente está en relación con la adherencia al tratamiento. La relación entre estas dos variables indica significancia estadística, el valor de p 0,033.

\section{Discusión}

Las estadísticas nacionales en relación al VIH/SIDA en Ecuador tienen un comportamiento de ascenso, tal es el caso que para el año 2017 existieron alrededor de 36.544 personas infectadas, con predominio en el grupo etareo considerado como sexualmente activo entre 15-49 años, y de estos el grupo más afectado fue el sexo masculino. Las provincias con el mayor número de personas infectadas son la provincia del Guayas en primer lugar y luego la provincia del Pichincha. El informe de monitoreo global de SIDA en Ecuador del año 2017, revela que en la provincia del Cañar existen 263 casos acumulados $(0.4 \%)$ de la población, para el año 2016 en Cañar reporta un total de 39 casos. $^{4}$

Como se menciona con anterioridad, en Cañar para el año 2016 se reportan 39 casos de VIH/SIDA. El estudio abarca un total de 32 pacientes que se atienden dentro de hospital Homero Castanier Crespo; así que por considerarse un grupo de población muy vulnerable es importante conocer la calidad de vida que tienen estos pacientes, sus hábitos nutricionales y la adherencia al tratamiento.

El estudio identificó variables sociodemográficas, observándose que el $56 \%$ de los pacientes están en una edad entre 30-39 años, existe un predominio del sexo masculino $(65,6 \%)$, residencia urbana $(59 \%)$, y el $49.6 \%$ tiene solo instrucción primaria, su orientación sexual en su mayoria $(84,4 \%)$ son heterosexuales, así mismo el informe GAM Ecuador en el año 2017 reporta que a nivel nacional existe un predominio del sexo masculino en los infectados, al igual que el grupo de edad más afectado corresponde a los pacientes que se encuentran entre los 15 y 49 años considerada como la edad sexualmente activa. Itzel y col. en México estudiaron la calidad de vida de los pacientes con diagnóstico de VIH/SIDA, y las características sociodemográficas de los pacientes mexicanos son muy simulares, se encontró que hubo predominio de pacientes con sexo masculino, la edad promedio estuvo en 30.8 años, su orientación sexual fue heterosexual mayoritariamente y en cuanto a su nivel de instrucción gran parte tenía un nivel de instrucción básico. ${ }^{10}$

La calidad de vida engloba una infinidad de características que contribuyen a mantener en la persona un nivel 
de satisfacción de manera que la persona pueda sentirse feliz dentro de la sociedad. Existen numerosos instrumentos validados para medir calidad de vida, sin embargo, resulta fundamental el disponer de instrumentos específicos para grupos vulnerables como es el caso de los pacientes con VIH. En este contexto para medir la calidad de vida de estos pacientes se utiliza la encuesta MOS-HIV SF30 que consta de 11 dimensiones. ${ }^{12}$

Las dimensiones de la encuesta son valoradas en base a rangos que van de 0.12 a 0.20 , la suma total de estos rangos determina la calidad de vida del paciente de tal forma que aquellos pacientes que tienen un score de 71 a 100 tienen una buena calidad de vida y aquellos cuyo score está por debajo de 71 tienen una mala calidad de vida. ${ }^{12}$

Los estudios analizados que constan como referencia del presente estudio se realizaron en base a la encuesta MOS-HIV SF 30. En relación a ello en Uruguay, Cabrera y colaboradores estudian los factores asociados a la calidad de vida de 101 pacientes, a los cuales se les aplicó el cuestionario MOS-HIV y cuestionario SMAQ para evaluar la adherencia al tratamiento. El score global de calidad de vida estuvo en $71.71 \pm 17.76$, score que determina una mala calidad de vida. ${ }^{12}$

En lo que se refiere a la adherencia al tratamiento, se relacionó con las dimensiones de la encuesta, pero no tuvo impacto con el score global. Respecto a ello en el estudio realizado en los pacientes del HHCC se encontró un score global de menos de 71 en el $53 \%$ de los pacientes y un score de más de 71 en el $46.9 \%$ de los pacientes. Referente a la adherencia de la misma forma se relacionó con la dimensión salud mental del paciente encontrándose significancia estadística p 0.033 , se relacionó con otras dimensiones y calidad de vida, igualmente no tuvo impacto sobre el score global. $^{12}$

La condición inmunológica de los pacientes con VIH/SIDA, exige a los pacientes adoptar conductas alimentarias saludables, el estudio identificó hábitos dietéticos, desayuno y equilibrio alimentario en base a la encuesta o test rápido de Krece Plus que fue aplicado a los pacientes. El mencionado test consta de 16 ítems, cada uno tiene una puntuación de más 1 o menos 1 . Siendo más 11 la máxima puntuación y menos 5 la mínima puntuación.

De acuerdo a la puntuación obtenida se determina como nivel nutricional alto, medio o bajo. En el grupo de estudio se evidenció que el $53.1 \%$ de los pacientes tuvo un vinel nutricional bajo, el score total fue menor a 5 . Se buscó relación y significancia estadística entre la variable calidad de vida y hábitos dietéticos del paciente mediante la prueba del Chi Cuadrado encontrándose que el valor de $\mathrm{p}=0,047$.

El score global de calidad de vida de los pacientes estudiados estuvo por debajo de 71 , lo cual significa que tienen una mala calidad de vida, al buscar relación y significancia estadística entre el score global de calidad de vida y calidad percibida por el paciente, que según la encuesta MOS-HIV SF 30 la determina como muy buena, buena, regular y mala, se obtuvo que mediante la prueba de Chi Cuadrado el valor de $\mathrm{p}=0,002$.

\section{Conclusiones}

El score global del cuestionario da como resultado una mala calidad de los pacientes, un alto porcentaje de pacientes no son adherentes al tratamiento, y según el test rápido para medir nivel nutricional, más de la mitad de los pacientes tiene un nivel nutricional bajo. Existe relación de significancia estadística entre las variables: calidad de vida y nivel nutricional, estado de salud general (calidad de vida) y salud general percibida, adherencia y salud mental del paciente.

\section{Recomendaciones}

La calidad de vida que tiene una persona es muy importante, más aún cuando se trata de un grupo vulnerable como lo son las personas que viven con VIH/SIDA, es importante la valoración de la calidad de vida desde una perspectiva intercultural, que permita valorar aspectos psicológicos, sociales, ambientales y espirituales de la vida.

\section{Fuente de Financiamiento}

Este estudio es autofinanciado

\section{Conflicto de Intereses}

No existen conflictos personales, profesionales, financieroso de otro tipo.

\section{Consentimiento Informado}

Voluntad de las personas para participar y firmar el consentimiento informado.

Se informó de forma clara y precisa la investigación a las personas participantes en el estudio.

Se tomaron precauciones y medidas para minimizar y prevenir los riesgos.

Se explicó que los datos personales serán totalmente protegidos, y se asegura el principio de confidencialidad de cada participante. Se mantuvo la confidencialidad del paciente utilizando las dos iniciales de primer y segundo nombre de igual forma del primer y segundo apellido.

\section{Referencias Bibliográficas}

1. Health-Related Quality of Life and Well. Foundation Health Measure Report; 2014.

2. MSP. Informe GAM Ecuador; 2017. Available from: Unaids.org.

3. VIH - Ministerio de Salud Pública; 2020. Available from: https://www.salud.gob.ec/vih.

4. Informe GAM-Ecuador (Monitoreo Global del Sida) ; 2017. Available from: https://www.unaids.org/ sites/default/files/country/documents/ECU_2017_ countryreport.pdf. 
5. Crispin Jenkinson. Quality of life. Encyclopædia Britannica, inc.; 2019. Available from: https://www.britannica. 1 com/topic/quality-of-life.

6. Constitución del Ecuador 2008; 2008. Available from: https://www.oas.org/juridico/mla/sp/ecu/sp_ecu-int-textconst.pdf.

7. Toda una Vida. Plan Nacional de Desarrollo 2017-2021; 2019. Available from: https://www.planificacion.gob.ec/ wp-content/uploads/downloads/2017/10/PNBV-26-OCTFINAL_0K.compressed1.pdf.

8. Cabrera Alonso SG, Arteta Dalchiele Z, Meré Rouco JJ, Cavalleri Ferrari F. Calidad de vida relacionada a la salud en personas con VIH y factores asociados. Revista Médica del Uruguay. 2018;34(1):3-41.

9. Frutos C, Aquino N, Denis A, Ferreira MD. Calidad de Vida en el Paciente con VIH en el Servicio de Clínica Médica, Hospital Central Instituto de Previsión Social Paraguay-2015. Revista Instituto Medico Tropica[Internet]. 2016;11(1):10-21.

10. Gutiérrez-Gabriel I, Godoy-Guinto J, Lucas-Alvarado H, Pineda-Germán B, Vázquez-Cruz E, Sosa-Jurado F, et al. Calidad de vida y variables psicológicas que afectan la adherencia al tratamiento anti-retroviral en pacientes mexicanos con infección por VIH/SIDA. Revista chilena de infectología. 2019;36(3):331-339.

. Achucarro S. Calidad de vida de pacientes con VIH/SIDA y atención. Revista del Instituto de Medicina Tropical. 2014;5(1):20-34.

12. Cabrera Alonso SG, Arteta Dalchiele Z, Meré Rouco JJ, Cavalleri Ferrari F. Calidad de vida relacionada a la salud en personas con VIH y factores asociados. Revista Médica del Uruguay. 2018;34(1):3-41.

Recibido: 10 de mayo de 2019

Aceptado: 25 de agosto de 2019 